\title{
Characterizations of Exponential Distribution Based on Two-Sided Random Shifts
}

\author{
Santanu Chakraborty and George P. Yanev \\ School of Mathematical and Statistical Sciences \\ The University of Texas Rio Grande Valley
}

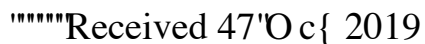

Accepted Q0() HEWD 2018

\begin{abstract}
A new characterization of the exponential distribution is obtained. It is based on an equation involving randomly shifted (translated) order statistics. No specific distribution is assumed for the shift random variables. The proof uses a recently developed technique including the Maclaurin series expansion of the probability density of the parent variable.

Keywords: characterization, exponential distribution, order statistics, random shifts
\end{abstract}

2010 MSC: 62G30, 62E10

\section{Introduction and main result}

Let $X_{1}, X_{2}, \ldots, X_{n}$ be a simple random sample from a continuously distributed parent $X$. Denote by $X_{j: n}$ for $1 \leq j \leq n$ the $j$ th order statistic (OS). Let $n_{1}, n_{2}, k_{1}$, and $k_{2}$ be fixed integers, such that $0 \leq k_{i} \leq n_{i}-1$ for $i=1,2$. Consider the distributional equation

$$
X_{n_{1}-k_{1}: n_{1}}+c_{1} \xi_{1} \stackrel{d}{=} X_{n_{2}-k_{2}: n_{2}}+c_{2} \xi_{2},
$$

where $c_{1}$ and $c_{2}$ are certain constants and the "shift" (or "translation") variables $\xi_{1}$ and $\xi_{2}$ are independent from $X_{1}, X_{2}, \ldots, X_{n}$. A variety of particular cases of (1) have appeared in a number of papers devoted to characterizations of certain classes of continuous distributions. Recent surveys can be found in [4] and [6].

If $c_{2}=0$, then (1) is called one-sided random shift equation. Let $k$ and $n$ be fixed integers, such that $1 \leq k \leq n-1$. Under some regularity conditions, 
it is proven in [3], [12] and [7] that each one of the following two equations characterizes the exponential distribution:

$$
\begin{gathered}
X_{n-k: n}+\frac{1}{k} \xi \stackrel{d}{=} X_{n-k+1: n} \quad \text { (consecutive OS) } \\
X_{n-k: n-1}+\frac{1}{n} \xi \stackrel{d}{=} X_{n-k+1: n} \quad \text { (consecutive OS and sample sizes) }
\end{gathered}
$$

where $\xi$ is unit exponential.

If both $c_{1}>0$ and $c_{2}>0$, then we have equation with to two-sided random shifts. It is established in [12] that for unit exponential $\xi_{1}$ and $\xi_{2}$, the equation

$$
X_{n-k: n-1}+\frac{1}{n} \xi_{1} \stackrel{d}{=} X_{n-k: n}+\frac{1}{k} \xi_{2},
$$

where $k$ and $n$ are fixed integers, such that $1 \leq k \leq n-1$, characterizes the exponential distribution.

In all equations above the random shifts are exponentially distributed and therefore (as the characterization implies) are also identically distributed with the parent variable $X$. In these notes the condition shifts variables to be exponential is dropped. Instead the weaker assumption that the random shifts are identically distributed with $X$ is made. The one-sided shift case without specifying the distribution of the shift variable is studied in [5] for $n=2$. Namely, it is proven that the equation

$$
X_{1}+\frac{1}{2} X_{2} \stackrel{d}{=} X_{2: 2}
$$

characterizes the exponential distribution. This is generalized in [8] and [13] where it is shown that for fixed $n \geq 2$ the equation

$$
X_{n-1: n-1}+\frac{1}{n} X_{n} \stackrel{d}{=} X_{n: n}
$$

characterizes the exponential distribution. It is worth mentioning that a similar characterization in [1] for a sample of size $n=2$ is based on the equation

$$
X_{1: 2}+X \stackrel{d}{=} X_{2: 2}
$$

In our main result below we obtain an analog of the two-sided characterization (2) dropping the assumption on the shifts to be exponential. In the proof 


\section{PRELIMINARIES}

of the theorem a recently developed technique based on the Maclaurin series expansion of the density function $f(x)$ is used. This method of proof grew out of an argument given first in [5].

Theorem Let $k$ and $n$ be fixed integers, such that $1 \leq k \leq n-1$. Let $X_{1}, X_{2}, \ldots, X_{n+1}$ be a simple random sample from a distribution with cdf $F(x)$ $(F(0)=0)$ and pdf $f(x)(f(0)>0)$. Assume that $f(x)$ is analytic for $x>0$. Then for some $\lambda>0$

$$
F(x)=1-e^{-\lambda x}, \quad x \geq 0
$$

if and only if

$$
X_{n-k: n-1}+\frac{1}{n} X_{n} \stackrel{d}{=} X_{n-k: n}+\frac{1}{k} X_{n+1} .
$$

In the next section, we present several lemmas needed to prove the theorem. In Section 3, we prove our main theorem.

\section{Preliminaries}

The first lemma plays a key role in the proof of the theorem. Originally it appeared in a similar form as an argument in [5]. In the form presented below, it was proven in [10].

Lemma 1 Let $f(x)$ be analytic for all $x>0$ and $f(0)>0$. If for all non-negative $m$

$$
f^{(m)}(0)=(-1)^{m} f^{m+1}(0),
$$

then

$$
f(x)=f(0) e^{-f(0) x} .
$$

Next, define for all non-negative integers $n$ and $i$, and any real $x$ the numbers

$$
H_{n, i}(x):=\sum_{j=0}^{n}(-1)^{j}\left(\begin{array}{l}
n \\
j
\end{array}\right)(x-j)^{i} .
$$

It is known (see [11]) that $H_{n, n}(\cdot)=n$ ! and $H_{n, i}(\cdot)=0$ for $0 \leq i \leq n-1$.

In the rest of the section we prove three more lemmas needed in the proof of the theorem. 


\section{PRELIMINARIES}

Lemma 2 For $m, n$ and $k$ positive integers with $1 \leq k \leq n$,

$$
\sum_{l=0}^{m}\left(\begin{array}{c}
m \\
l
\end{array}\right) H_{n-k+l, i}(n-k+l+1)=H_{n-k, i}(n-k+m+1)
$$

Proof. Let $s$ and $r$ be positive integers. We shall prove that

$$
H_{s, r}(s+1)+H_{s+1, r}(s+2)=H_{s, r}(s+2) .
$$

Using the identity

$$
\left(\begin{array}{c}
s+1 \\
j
\end{array}\right)-\left(\begin{array}{c}
s \\
j-1
\end{array}\right)=\left(\begin{array}{l}
s \\
j
\end{array}\right)
$$

and the definition of $H_{i, j}(x)$, we obtain for $r \geq s+1$

$$
\begin{aligned}
H_{s, r}(s+1)+H_{s+1, r}(s+2) \\
\quad=\sum_{j=0}^{s}(-1)^{j}\left(\begin{array}{c}
s \\
j
\end{array}\right)(s+1-j)^{r}+\sum_{j=0}^{s+1}(-1)^{j}\left(\begin{array}{c}
s+1 \\
j
\end{array}\right)(s+2-j)^{r} \\
=\sum_{j=1}^{s+1}\left[(-1)^{j-1}\left(\begin{array}{c}
s \\
j-1
\end{array}\right)+(-1)^{j}\left(\begin{array}{c}
s+1 \\
j
\end{array}\right)\right](s+2-j)^{t}+(s+2)^{r} \\
=\sum_{j=0}^{s+1}(-1)^{j}\left(\begin{array}{c}
s \\
j
\end{array}\right)(s+2-j)^{r} \\
=H_{s, r}(s+2) .
\end{aligned}
$$

It follows from (8) that

$$
H_{n-k, i}(n-k+1)+H_{n-k+1, i}(n-k+2)=H_{n-k, i}(n-k+2)
$$

Proceeding one more step, we have,

$H_{n-k, i}(n-k+1)+2 H_{n-k+1, i}(n-k+2)+H_{n-k+2, i}(n-k+3)=H_{n-k, i}(n-k+3)$

Suppose the lemma is true for $m \leq r$. We shall prove it for $m=r+1$. Indeed,

$$
\begin{aligned}
& \sum_{l=0}^{r+1}\left(\begin{array}{c}
r+1 \\
l
\end{array}\right) H_{n-k, l}(n-k+l+1) \\
& \quad=\sum_{l=0}^{r}\left(\begin{array}{l}
r \\
l
\end{array}\right) H_{n-k+l, i}(n-k+l+1)+\sum_{l=0}^{r}\left(\begin{array}{l}
r \\
l
\end{array}\right) H_{n-k+l+1, i}(n-k+l+2) \\
& \quad=H_{n-k, i}(n-k+r+1)+H_{n-k+1, i}(n-k+r+2)=H_{n-k, i}(n-k+r+2) .
\end{aligned}
$$




\section{PRELIMINARIES}

The proof is complete.

Lemma 3 Let $k, n$, and $r$ be positive integers such that $1 \leq k \leq n-1$. Denote $t=n-k-1$. The following two identities are true:

$$
\sum_{i=0}^{r} k^{i} H_{t, t+r+1-i}(n)=\frac{1}{t+1} H_{t+1, t+r+2}(n)-k^{r+1} t !
$$

and

$$
\sum_{i=0}^{r} n^{i} H_{t, t+r+1-i}(n-1)=\frac{1}{t+1} H_{t+1, t+r+2}(n)-n^{r+1} t ! .
$$

Proof. We shall prove (9). The proof of (10) is similar. We have

$$
\begin{aligned}
& \sum_{i=0}^{r} k^{i} H_{t, t+r+1-i}(n)=\sum_{i=0}^{r} k^{i} \sum_{j=0}^{t}(-1)^{j}\left(\begin{array}{l}
t \\
j
\end{array}\right)(n-j)^{t+r+1-i} \\
& =\sum_{j=0}^{t}(-1)^{j}\left(\begin{array}{l}
t \\
j
\end{array}\right) \sum_{i=0}^{r} k^{i}(n-j)^{t+r+1-i} \\
& =\sum_{j=0}^{t}(-1)^{j}\left(\begin{array}{l}
t \\
j
\end{array}\right)(n-j)^{t+r+1} \sum_{i=0}^{r}\left(\frac{k}{n-j}\right)^{i} \\
& =\sum_{j=0}^{t}(-1)^{j}\left(\begin{array}{l}
t \\
j
\end{array}\right)(n-j)^{t+r+1}\left[\frac{1-\left(\frac{k}{n-j}\right)^{r+1}}{1-\frac{k}{n-j}}\right] \\
& =\sum_{j=0}^{t}(-1)^{j}\left(\begin{array}{l}
t \\
j
\end{array}\right) \frac{(n-j)^{t+r+2}}{t+1-j}\left[1-\left(\frac{k}{n-j}\right)^{r+1}\right] \\
& =\frac{1}{t+1} \sum_{j=0}^{t}(-1)^{j}\left(\begin{array}{c}
t+1 \\
j
\end{array}\right)(n-j)^{t+r+2}\left[1-\left(\frac{k}{n-j}\right)^{r+1}\right] \\
& =\frac{1}{t+1} \sum_{j=0}^{t}(-1)^{j}\left(\begin{array}{c}
t+1 \\
j
\end{array}\right)(n-j)^{t+r+2}-\frac{k^{r+1}}{t+1} \sum_{j=0}^{t}(-1)^{j}\left(\begin{array}{c}
t+1 \\
j
\end{array}\right)(n-j)^{t+1} \\
& =\frac{1}{t+1} \sum_{j=0}^{t+1}(-1)^{j}\left(\begin{array}{c}
t+1 \\
j
\end{array}\right)(n-j)^{t+r+2}-\frac{k^{r+1}}{t+1} \sum_{j=0}^{t+1}(-1)^{j}\left(\begin{array}{c}
t+1 \\
j
\end{array}\right)(n-j)^{t+1} \\
& =\frac{1}{t+1} H_{t+1, t+r+2}(n)-\frac{k^{r+1}}{t+1} H_{t+1, t+1}(n) \\
& =\frac{1}{t+1} H_{t+1, t+r+2}(n)-k^{r+1} t \text {. }
\end{aligned}
$$

Lemma 4 Let $j \geq 1$ and $d$ be integers, such that $j+d \geq 0$. Assume $F(0)=0$ 


\section{PRELIMINARIES}

and for $d \geq 1$

$$
f^{(m)}(0)=(-1)^{m} f^{m+1}(0), \quad m=1,2, \ldots, d .
$$

Then for $j=1,2, \ldots$

$$
G_{j}^{(j+d)}(0)=\left\{\begin{array}{llr}
H_{j, j+d}(j+1) f^{j+1-d}(0)\left(f^{\prime}(0)\right)^{d} & \text { if } & d \geq 0 \\
0 & \text { if } & -j \leq d<0 .
\end{array}\right.
$$

where $G_{j}(x):=F^{j}(x) f(x)$.

Proof. (i) If $-j \leq d<0$, then $G_{j}^{(j+d)}(0)=0$ because all the terms in the expansion of $G_{j}^{(j+d)}(0)$ have a factor $F(0)=0$.

(ii) Let $d=0$. We shall prove (13) by induction on $j$. One can verify directly the case $j=1$. Assuming (13) for $j=k$, we shall prove it for $j=k+1$. Since $G_{k+1}(x)=F(x) G_{k}(x)$, applying (i), we see that

$$
\begin{aligned}
& G_{k+1}^{(k+1)}(0)=\sum_{i=0}^{k+1}\left(\begin{array}{c}
k+1 \\
i
\end{array}\right) F^{(i)}(0) G_{k}^{(k+1-i)}(0) \\
& \quad=F(0) G_{k}^{(k+1)}(0)+(k+1) F^{\prime}(0) G_{k}^{(k)}(0)+\sum_{i=2}^{k+1}\left(\begin{array}{c}
k+1 \\
i
\end{array}\right) F^{(i)}(0) G_{k}^{(k+1-i)}(0) \\
& \quad=(k+1) ! f^{k+2}(0)
\end{aligned}
$$

which completes the proof of (ii).

(iii) Let $d>0$ and $j$ be any positive integer. For simplicity, we will write $f^{(i)}:=f^{(i)}(0)$ below.

(a) Let $j=1$. If $d=1$, then we have $G_{1}^{(2)}(0)=3 f^{\prime} f=f^{\prime} f H_{1,2}(2)$ since $H_{1,2}(2)=3$. Thus, (13) is true for $d=1$. Next, assuming (13) for $G_{1}^{(k)}(0)$, we shall prove it for $G_{1}^{(k+1)}(0)$. Since $G_{1}(x)=F(x) f(x)$, using (12) we obtain

$$
\begin{aligned}
G_{1}^{(k+1)}(0) & =\sum_{i=1}^{k+1}\left(\begin{array}{c}
k+1 \\
i
\end{array}\right) f^{(i-1)} f^{(k+1-i)} \\
& =\sum_{i=1}^{k+1}\left(\begin{array}{c}
k+1 \\
i
\end{array}\right)(-1)^{i-1} f^{i}(-1)^{k+1-i} f^{k+2-i} \\
& =(-1) f^{k+2} \sum_{j=1}^{k+1}\left(\begin{array}{c}
k+1 \\
j
\end{array}\right) \\
& =(-1) f^{k+2} H_{1,1+k}(2)
\end{aligned}
$$




\section{PRELIMINARIES}

This completes the proof for the case (a) $j=1$ and any $d>0$.

(b) Assuming (13) for $j=1,2, \ldots k$ and any $d>0$ we shall prove it for $j=k+1$ and any $d>0$. Since $G_{k+1}(x)=F(x) G_{k}(x)$, by (12) and the induction assumption, we obtain

$$
\begin{aligned}
G_{k+1}^{(k+1+d)}(0) & =\sum_{i=1}^{k+1+d}\left(\begin{array}{c}
k+1+d \\
i
\end{array}\right) f^{(i-1)} G_{k}^{(k+1+d-i)}(0) \\
& =\sum_{i=1}^{d+1}\left(\begin{array}{c}
k+1+d \\
i
\end{array}\right) f^{(i-1)} G_{k}^{(k+1+d-i)}(0) \\
& =\sum_{i=1}^{d+1}\left(\begin{array}{c}
k+1+d \\
i
\end{array}\right)(-1)^{i-1} f^{i} f^{k-d+i}\left(f^{\prime}\right)^{1+d-i} H_{k, k+1+d-j}(k+1) \\
& =f^{k+2-d}\left(f^{\prime}\right)^{d} \sum_{i=1}^{k+1+d}\left(\begin{array}{c}
k+1+d \\
i
\end{array}\right) H_{k, k+1+d-i}(k+1) \\
& =f^{k+2-d}\left(f^{\prime}\right)^{d} \sum_{l=0}^{k+d}\left(\begin{array}{c}
k+1+d \\
l
\end{array}\right) H_{k, l}(k+1),
\end{aligned}
$$

where in the last equality we have made the index change $l=k+1+d-j$. Therefore, to finish the proof of the induction step (b), we need to show that

$$
\sum_{l=0}^{k+d}\left(\begin{array}{c}
k+1+d \\
l
\end{array}\right) H_{k, l}(k+1)=H_{k+1, k+1+d}(k+2) .
$$

For brevity denote $r=k+1+d$. Using the definition of $H_{k, l}(k+1)$, we obtain

$$
\begin{aligned}
\sum_{l=0}^{r-1}\left(\begin{array}{l}
r \\
l
\end{array}\right) H_{k, l}(k+1)=\sum_{i=0}^{k}(-1)^{i}\left(\begin{array}{l}
k \\
i
\end{array}\right) \sum_{l=0}^{r-1}\left(\begin{array}{l}
r \\
l
\end{array}\right)(k+1-i)^{l} \\
=\sum_{i=0}^{k}(-1)^{i}\left(\begin{array}{c}
k \\
i
\end{array}\right)\left[(k-i+2)^{r}-(k-i+1)^{r}\right] \\
=(k+2)^{r}-\left[(k+1)^{r}+\left(\begin{array}{c}
k \\
1
\end{array}\right)(k+1)^{r}\right]+\ldots \\
\quad+(-1)^{k}\left[\left(\begin{array}{c}
k \\
k-1
\end{array}\right) 2^{r}+2^{r}\right]+(-1)^{k+1} \\
=(k+2)^{r}-\left(\begin{array}{c}
k+1 \\
1
\end{array}\right)(k+1)^{r}+\ldots+(-1)^{k}\left(\begin{array}{c}
k+1 \\
k
\end{array}\right) 2^{r}+(-1)^{k+1} \\
=\sum_{j=0}^{k+1}(-1)^{j}\left(\begin{array}{c}
k+1 \\
j
\end{array}\right)(k+2-j)^{r} \\
=H_{k+1, k+1+d}(k+2) .
\end{aligned}
$$




\section{PROOF OF THE THEOREM}

This proves the induction step (b). Now (iii) follows from (a) and (b). The proof of the lemma is complete.

\section{Proof of the Theorem}

It is not difficult to see that (4) is equivalent to (for brevity $t:=n-k-1$ )

$$
\int_{0}^{x} F^{t}(u)(1-F(u))^{k-1} f(u) f(n(x-u)) d u=\int_{0}^{x} F^{t}(u)(1-F(u))^{k} f(u) f(k(x-u)) d u .
$$

Recalling from Lemma 4 that $G_{j}(x):=F^{j}(x) f(x)$ for $j=1,2, \ldots$ and using the binomial formula, we write last equation as

$$
\begin{aligned}
\int_{0}^{x} \sum_{l=0}^{k-1}\left(\begin{array}{c}
k-1 \\
l
\end{array}\right) & (-1)^{l} G_{t+l}(u) f(n(x-u)) d u \\
& =\int_{0}^{x} \sum_{l=0}^{k}\left(\begin{array}{c}
k \\
l
\end{array}\right)(-1)^{l} G_{t+l}(u) f(k(x-u)) d u
\end{aligned}
$$

Differentiating with respect to $x$ as many as $t+r+2$ times for $r \geq 0$ and substituting $x=0$, we obtain

$$
\begin{aligned}
\sum_{l=0}^{k}(-1)^{l}\left(\begin{array}{c}
k-1 \\
l
\end{array}\right) & \sum_{i=0}^{t+r+1} n^{i} G_{t+l}^{(t+r+1-i)}(0) f^{(i)}(0) \\
& =\sum_{l=0}^{k}(-1)^{l}\left(\begin{array}{c}
k \\
l
\end{array}\right) \sum_{i=0}^{t+r+1} k^{i} G_{t+l}^{(t+r+1-i)}(0) f^{(i)}(0) .
\end{aligned}
$$

In view of Lemma 1, to prove that (15) implies (6), it is sufficient to show that it implies (5). We shall prove (5) by induction with respect to $m$. Let us first verify (5) for $m=1$, i.e., $f^{\prime}(0)=-f^{2}(0)$. If $r=0$ then (15) becomes

$$
\begin{aligned}
\sum_{l=0}^{k}(-1)^{l}\left(\begin{array}{c}
k-1 \\
l
\end{array}\right) \sum_{i=0}^{t+1} n^{i} G_{t+l}^{(t+1-i)}(0) f^{(i)}(0) \\
=\sum_{l=0}^{k}(-1)^{l}\left(\begin{array}{l}
k \\
l
\end{array}\right) \sum_{i=0}^{t+1} k^{i} G_{t+l}^{(t+1-i)}(0) f^{(i)}(0) .
\end{aligned}
$$

Since, by Lemma $4, G_{t+l}^{(t+1-i)}=0$ when $t+1-i<t+l$, omitting the zero terms in the sums and simplifying we obtain

$$
(t+1) G_{t}^{(t)}(0) f^{\prime}(0)=-G_{t+1}^{(t+1)}(0) f(0),
$$




\section{PROOF OF THE THEOREM}

which, in view of Lemma 4 , is equivalent to

$$
(t+1) f^{t+1}(0) t ! f^{\prime}(0)=-f^{t+2}(0)(t+1) ! f(0)
$$

and thus $f^{\prime}(0)=-f^{2}(0)$. Thus, (5) is true for $m=1$.

To prove the induction step suppose (5) holds for $m=1,2, \ldots, r$, i.e.,

$$
f^{(m)}(0)=(-1)^{m} f^{m+1}(0), \quad m=1,2, \ldots, r .
$$

We shall prove it for $m=r+1$. Under the induction hypothesis (17), Lemma 2 implies $G_{t+l}^{(t+r+1-i)}(0)>0$ only if $i \leq r-l+1$ and thus, omitting the zero terms in the sums of (15), we have

$$
\begin{aligned}
\sum_{l=0}^{k}(-1)^{l}\left(\begin{array}{c}
k-1 \\
l
\end{array}\right) & \sum_{i=0}^{r-l+1} n^{i} G_{t+l}^{(t+r+1-i)}(0) f^{(i)}(0) \\
& =\sum_{l=0}^{k}(-1)^{l}\left(\begin{array}{c}
k \\
l
\end{array}\right) \sum_{i=0}^{r-l+1} k^{i} G_{t+l}^{(t+r+1-i)}(0) f^{(i)}(0)
\end{aligned}
$$

and interchanging the sums, we write it as

$$
\begin{aligned}
& \sum_{i=0}^{r+1} n^{i} f^{(i)}(0) \sum_{l=0}^{r-i+1}(-1)^{l}\left(\begin{array}{c}
k-1 \\
l
\end{array}\right) G_{t+l}^{(t+r+1-i)}(0) \\
&=\sum_{i=0}^{r+1} k^{i} f^{(i)}(0) \sum_{l=0}^{r-i+1}(-1)^{l}\left(\begin{array}{l}
k \\
l
\end{array}\right) G_{t+l}^{(t+r+1-i)}(0) .
\end{aligned}
$$

Collecting in the left-hand side the terms with $i=r+1$, results in

$$
\begin{aligned}
\left(n^{r+1}\right. & \left.-k^{r+1}\right) f^{(r+1)}(0) G_{t}^{(t)}(0) \\
& =\sum_{i=0}^{r} f^{(i)}(0) \sum_{l=0}^{r-i+1}\left[k^{i}\left(\begin{array}{c}
k \\
l
\end{array}\right)-n^{i}\left(\begin{array}{c}
k-1 \\
l
\end{array}\right)\right](-1)^{l} G_{t+l}^{(t+r+1-i)}(0) .
\end{aligned}
$$

Applying Lemma 4 to both sides of (18) and taking into account the induction hypothesis (17) in the right-hand side, it is not difficult to see that

$$
\begin{aligned}
& \left(n^{r+1}-k^{r+1}\right) f^{(r+1)}(0) t ! f^{t+1}(0) \\
& \quad=(-1)^{r+1} f^{t+r+3}(0) \sum_{i=0}^{r} \sum_{l=0}^{k}\left[k^{i}\left(\begin{array}{c}
k \\
l
\end{array}\right)-n^{i}\left(\begin{array}{c}
k-1 \\
l
\end{array}\right)\right] H_{t+l, t+r+1-i}(t+1+l),
\end{aligned}
$$


where $\left(\begin{array}{c}p-1 \\ k\end{array}\right)=0$. Now, it is clear that proving (5) for $m=r+1$ is equivalent to proving

$$
\left(n^{r+1}-k^{r+1}\right) t !=\sum_{i=0}^{r} \sum_{l=0}^{k}\left[k^{i}\left(\begin{array}{l}
k \\
l
\end{array}\right)-n^{i}\left(\begin{array}{c}
k-1 \\
l
\end{array}\right)\right] H_{t+l, t+r+1-i}(t+1+l) .
$$

Applying Lemma 2 we write last equation as

$$
\left(n^{r+1}-k^{r+1}\right) t !=\sum_{i=0}^{r} k^{i} H_{t, t+r+1-i}(n)-\sum_{i=0}^{r} n^{i} H_{t, t+r+1-i}(n-1)
$$

Finally, it is easily to see that (19) with $m=r+1$ follows from (9) and (10) in Lemma 3. This proves (5) for $m=r+1$, which in turn completes the induction argument and theorem's proof.

\section{References}

[1] Ahsanullah, M. and Anis, M.Z., 2017: Some Characterizations of Exponential Distributions, International Journal of Statistics and Probability, 6(5), 132 - 139.

[2] Ahsanullah, M. and Hamedani, G. G., Exponential Distribution, 2010: Theory and Methods, NOVA Science, New York.

[3] Alzaid, A.A. and Ahsanullah, M. 2003: A characterization of the Gumbel distribution based on record values, Comm. Stats. Theory and Methods, $32,11: 2101-2108$.

[4] Ananjevskii, S.M. and Nevzorov, V.B., 2016: On families of distributions which are characterized by some properties of ordered random variables, Vestnik of Saint Petersburg University. Series 1, Mathematics. Mechanics. Astronomy, Vol. 3(61), 345-354. In Russian (English summary).

[5] Arnold, B.C. and Villaseñor, J.A., 2013: Exponential Characterizations Motivated by the Structure of Order Statistics in Sample of Size Two, Statistics and Probability. Letters 83, 596-601. 
[6] Berred, A. and Nevzorov, V.B., 2013: Characterizations of families of distributions, which include the logistic one, by properties of order statistics. J. Math. Sci., 188, 6:673-676.

[7] Castaño-Martinez, A., López-Blázquez, F., Salamanea-Miño, B. Random translations, 2012: Contractions and dilations of order statistics and records. Statistics, 46, 57-67.

[8] Chakraborty, S. and Yanev, G.P., 2013: Characterization of exponential distribution through equidistribution conditions for consecutive maxima, J. Statist. Appl. \& Probab. 2, 237-242.

[9] Conway, J. B., 1978: Functions of One Complex Variable (Graduate Texts in Mathematics - Vol 11) (v. 1) 2nd Ed., Springer, New York, USA.

[10] Obradović, M., 2015: Three Characterizations of Exponential Distribution Involving Median of Sample of Size Three. J. Statistical Theory and Appl., $14,3: 257-264$.

[11] Ruiz, S.M., 1996: An algebraic identity leading to Wilson's theorem. The Math. Gazette 80, 579-582.

[12] Wesolowski, J. and Ahsanullah, M., 2004: Switching order statistics through random power contractions. Aust. N. Z. J. Statist. 46, 297-303.

[13] Yanev, G.P. and Chakraborty, S., 2016: A characterization of exponential distribution and the SukhatmeRnyi decomposition of exponential maxima, Statistics and Probability Letters, 110, 94 - 102. 